\title{
Baryon-(anti-)baryon interaction cross-section measurement with femtoscopy technique in HIC
}

\author{
Adam Kisiel ${ }^{* \dagger}$ \\ Warsaw University of Technology, Faculty of Physics \\ Koszykowa 75, 00-662 Warsaw, Poland \\ E-mail: kisieleif.pw.edu.pl
}

\begin{abstract}
Interaction cross-sections for baryon pairs are of fundamental interest and they are actively investigated theoretically. They are well known for pairs of common (anti-)baryons; however, there is a lack of precise data for heavier baryons, including the ones carrying strangeness. The twoparticle correlation formalism (femtoscopy) is sensitive to the interaction kernel for a pair of particles, which is related to the pair interaction cross-section. This formalism is extensively used to measure two-particle correlations in heavy-ion collisions. In particular, the collisions at RHIC and LHC produce simultaneously a large number of baryons and anti-baryons. We show how this formalism can be used to extract the cross-sections from the femtoscopic baryon-(anti-) baryon correlation functions. The analysis is complicated by the presence of the so-called "residual correlations" arising from weak decay products in the measured sample. We show how this effect can be exploited to gain further insight into the cross-sections of even heavier baryons. We discuss the limitations of the measurement technique and estimate the discovery potential of currently available and soon-to-be-collected heavy-ion collision datasets at RHIC and at the LHC.
\end{abstract}

XVII International Conference on Hadron Spectroscopy and Structure - Hadron2017

25-29 September, 2017

University of Salamanca, Salamanca, Spain

\footnotetext{
* Speaker.

$\dagger$ This work is supported by Polish National Science Centre under grant No. UMO-2014/13/B/ST2/04054 and UMO-2016/22/M/ST2/00176.
} 


\section{Introduction}

Interaction of baryons is a fundamental subject in nuclear physics. It is being studied by various methods, including detailed analysis of the stable and unstable nuclei as well as dedicated scattering experiments. The knowledge is then used in a wide array of fields, such as baryogenesis in the early Universe, the study of the neutron stars [1,2] and the evolution of the heavy-ion collisions [3]. At the moment, interaction of baryons is well known for protons, neutrons and their antiparticles [4]. Data also exist for interaction with deuterons. However, the interaction involving baryons with strangeness is less understood. Data exist on $\Sigma^{-} p$ interaction for centerof-mass energy $(\sqrt{s})$ above $5 \mathrm{GeV}$ and for $p \Lambda$ interaction $[5,6,7]$. The existing experimental measurements are of limited quality. Data on interaction between baryons and antibaryons with non-zero strangeness is even more limited. As a result, commonly used hadronic rescattering models, such as UrQMD, assume the annihilation between all baryon-antibaryon pairs has the same cross-section as the proton-antiproton interaction, when taken at the same $\sqrt{s}$ [3]. This process for $p \bar{p}$ was studied in great detail theoretically $[8,9,10,11]$. Some models aim to describe the baryon interaction via meson-exchange interaction [12, 13, 14], others use the Chiral Effective Theory [15]. Both of these approaches would benefit greatly from constraints coming from new experimental data. Especially information on the behaviour of the cross-section at low relative momentum, where it is large and changes rapidly, would be desirable.

Heavy-ion collisions at ultra-relativistic energies are studied extensively at Relativistic HeavyIon Collider (RHIC) at $\sqrt{s_{N N}}=200 \mathrm{GeV}$ and at the Large Hadron Collider (LHC) at $2.76 \mathrm{TeV}$ and 5.02 TeV. In the collisions of $\mathrm{Au}$ or Pb nuclei a large system of deconfined Quark-Gluon Plasma (QGP) is created. It expands rapidly, cools down and reaches a state when quarks must recombine back into hadrons. This process is well described by a statistical model. The baryochemical potential of the system is close to zero, which means that the number of produced baryons and antibaryons is very similar $[16,17]$. As a result a significant number of baryons and antibaryons are produced simultaneously in each event. This in turn means that a large number of baryon-baryon and baryon-antibaryon pairs can be considered. The majority the baryons is emitted with relatively small transverse momentum $p_{\mathrm{T}}$, below $3 \mathrm{GeV} / c$. The relative momentum, which determines also the $\sqrt{s}$ in the rest frame of the pairs, is therefore also limited. This presents a unique opportunity for experimental study of many types of baryon-(anti-)baryon pairs, including those with strange baryons, at low relative momentum.

The method which is best suited for this task is "femtoscopy", or the study of two-particle correlations as a function of pair relative momentum magnitude. It requires that the experiment performs the particle identification (PID) on a particle-by-particle basis at the low transverse momentum. The STAR detector at RHIC and the ALICE detector at the LHC have such capabilities. In this manuscript we will describe briefly the femtoscopic method, introduce the concept of "residual correlations" and discuss the experimental opportunities in STAR and ALICE.

\section{Femtoscopy method}

Experimentally, the femtoscopic correlation function is constructed for pairs of particles, where each particle is of a given type (for example a proton and a $\Lambda$ ). A distribution $A$ of the relative mo- 
menta of such pairs (more precisely of the magnitude of momentum of the "first" particle in the pair in the rest frame of the pair, denoted as $k^{*}$ ) is created for all pairs where both particles are produced in the same event. In order to account for trivial single particle acceptance similar distribution $B$ is constructed where the two particles in the pair come from two different events. The correlation function is then

$$
C\left(k^{*}\right)=A\left(k^{*}\right) / B\left(k^{*}\right) .
$$

$C$ is interpreted with the integral equation:

$$
C\left(k^{*}\right)=\int S\left(\mathbf{r}, \overrightarrow{k^{*}}\right)\left|\Psi\left(\mathbf{r}, \vec{k}^{*}\right)\right|^{2} \mathrm{~d}^{4} r \mathrm{~d} \cos (\theta) \mathrm{d} \phi,
$$

where $S$ describes a probability to emit a pair of particles of the given type from the source from points separated by $\mathbf{r}$ with a given relative momentum $k^{*}$, while $\Psi$ describes the interaction for a given pair type. The integration is also done over all angles of $k^{*}$. For non-identical baryons, when at least one of them is not charged the interaction can be described by a scattering on the strong interaction potential, taken with the inverse time direction [18]:

$$
\Psi\left(\mathbf{r}, \vec{k}^{*}\right)=\exp \left(-\overrightarrow{k^{*}} \vec{r}\right)+f^{S} \frac{\exp \left(i k^{*} r\right)}{r}
$$

where $f^{S}$ is the scattering amplitude, dependent on pair spin. In the s-wave approximation it can be expressed as:

$$
f^{S}=\left(\frac{1}{f_{0}}+\frac{1}{2} d_{0} k^{* 2}-i k^{*}\right)^{-1}
$$

The parameter $f_{0}$, the scattering length, is a complex number. Its magnitude determines the crosssection at low $k^{*}$ and its imaginary part described the annihilation channel. Parameter $d_{0}$ is the effective range of the potential.

In heavy-ion collisions $S$ is assumed to be a three-dimensional ellipsoid with Gaussian density distribution, characterized by the overall size $R$. Then the integral in Eq. (2.2) for the strong interaction gives $[19,18]$ :

$$
C\left(k^{*}\right)=1+\sum_{S} \rho_{S}\left[\frac{1}{2}\left|\frac{f^{S}\left(k^{*}\right)}{R}\right|^{2}\left(1-\frac{d_{0}^{S}}{2 \sqrt{\pi} R}\right)+\frac{2 \Re f^{S}\left(k^{*}\right)}{\sqrt{\pi} R} F_{1}\left(2 k^{*} R\right)-\frac{\mathfrak{J} f^{S}\left(k^{*}\right)}{R} F_{2}\left(2 k^{*} R\right)\right],
$$

where $F_{1}$ and $F_{2}$ are known functions, $S$ denotes possible pair spin orientations and $\rho_{S}$ gives the spin fractions. This formula can be directly fitted to correlations measured according to Eq. (2.1). In Eq. (2.5) the scattering amplitude $f^{S}$ and its components are usually divided by system size $R$. This means that by changing both in a similar direction one obtains very similar values of the correlation. In other words an attempt to fit those two parameters together will most likely result in a degenerate fit. Therefore, realistically one should fix one and fit another. For baryon pairs, $R$ can often be strongly constrained by femtoscopic measurements of other pair types. Then the scattering parameters $f_{0}$ and $d_{0}$ can be extracted from the fit. That is the basis of the methodology to extract the strong interaction potential parameters at low relative momentum from the analysis of the two-baryon femtoscopic correlation function measured in ultra-relativistic heavy-ion collisions. 


\section{Residual correlations}

Baryons interact via the strong interaction on a timescale of the order of tens of $\mathrm{fm} / \mathrm{c}$. Hyperons and multi-strange baryons decay on a weak interaction timescale, which is orders of magnitude larger. In a collider setup (for example in STAR and ALICE) a daughter baryon often travels in a direction very similar to the parent baryon, due to its large mass in comparison to the decay momentum. As a result it may be difficult to distinguish, in a sample of measured baryons, which are "primary" and which are decay products. In the correlation function one than has pairs which are composed of primary particles and others in which at least one baryon comes from the decay. For a "decay" pair the original correlation, described by Eq. (2.3), comes from the parent pair. Even though it is smeared by the decay, it can still have significant influence on the overall correlation. This is called "residual correlation". It is important if three conditions are met simultaneously [20]: (A) the parent correlation is strong, (B) a significant fraction of measured baryons comes from the given decay channel, (C) the decay momentum is small with respect to the width of the parent correlation effect. All those conditions are met for baryon pairs; therefore, residual correlations are important and must be properly taken into account. The work [20] provides a formalism in which such correlations can be incorporated into the analysis using the so-called transformation matrices, and pair fractions giving the contribution of each type of parent pair to the final sample of measured pairs. The correlation can be described as

$$
C\left(k_{A B}^{*}\right)=1+\sum_{X Y} \lambda_{X Y} \frac{\int C_{X Y}\left(k_{X Y}^{*}\right) T\left(k_{X Y}^{*}, k_{A B}^{*}\right) \mathrm{d} k_{X Y}^{*}}{\int T\left(k_{X Y}^{*}, k_{A B}^{*}\right) \mathrm{d} k_{X Y}^{*}},
$$

where $A$ and $B$ are baryon types that compose the final measured pair, $X$ and $Y$ are heavier baryons that decay weakly into $A$ and $B$, respectively. Parameters $\lambda$ are the pair fractions and $T$ is the transformation matrix, describing the probability that a parent pair with $k_{X Y}^{*}$ decays into the daughter pair with $k_{A B}^{*}$. The shape of $T$ depends strongly on $X$ and $Y$ decay kinematics and usually has the form of a diagonal band with the width roughly equal to the sum of decay momenta.

\section{Measurement potential}

STAR and ALICE have shown the capability to measure and identify on a track-by-track basis protons, $\Lambda, \Xi$, and $\Omega$ baryons. Out of those, protons and $\Lambda$ 's are produced with large perevent multiplicities. Therefore even with the current dataset it should be possible to measure the correlation functions for $p p, p \bar{p}, \bar{p} \bar{p}, p \Lambda, p \bar{\Lambda}, \bar{\Lambda} \bar{p}, \bar{\Lambda} p, \Lambda \Lambda, \Lambda \bar{\Lambda}$, and $\bar{\Lambda} \bar{\Lambda}$ pairs. The ALICE Collaboration has recently shown preliminary results for all these correlations in several centrality intervals. It is estimated that for example for $p p$ pairs the fraction of residual $p \Lambda$ pairs is of the same order as the fraction primary pairs. Clearly the analysis must then be performed using the formalism given in Eq. (3.1).

Figure 1 illustrates the inter-dependencies between various baryon pairs. The pair types in solid circles are the one that can be measured in STAR and ALICE at the moment. The ones in dashed circles can be potentially measured in the next LHC run. Most residual pairs contribute to only one of the three measured pair types. But four of them contribute simultaneously to two. In addition the pairs measured directly are also a residual contribution to other measured pairs. 


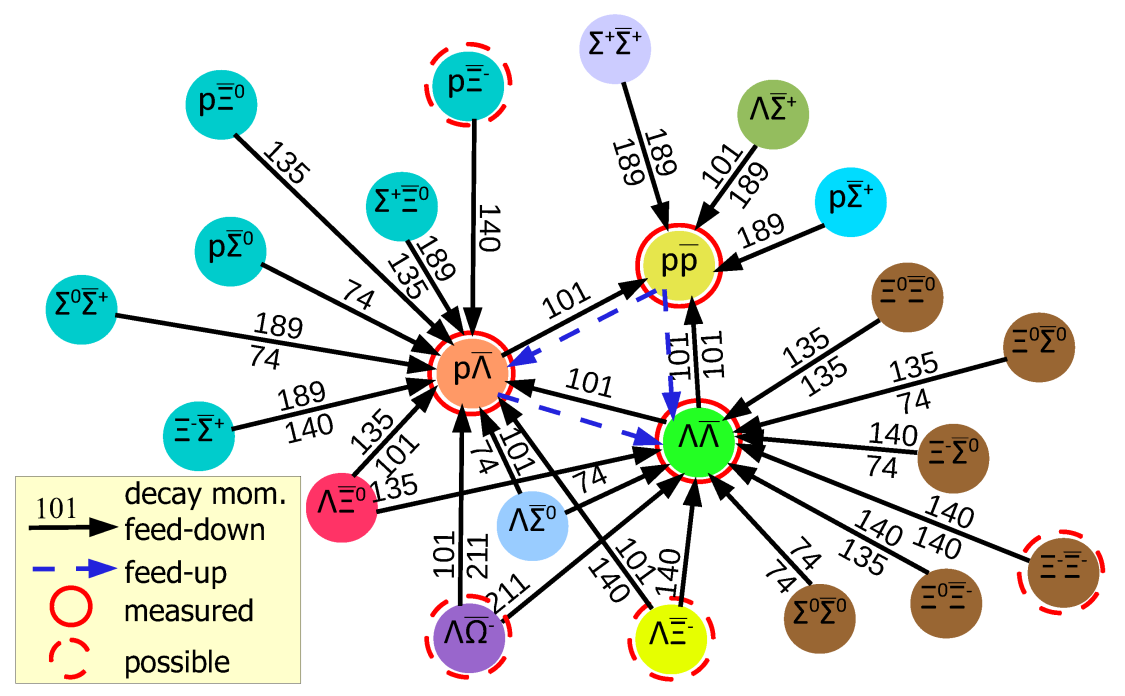

Figure 1: Residual components interlinks, with decay momenta specified for each decay (in $\mathrm{MeV} / \mathrm{c}$ (two momenta are given if both particles in the pair decay).

Moreover, the $\Lambda$ baryons are reconstructed from a combination of a pion and a proton track in the detector. It may happen that a random association of a proton and a pion appears as a $\Lambda$. If this "fake" $\Lambda$ is correlated with a proton, the correlation between this proton and the one contributing to the "fake" $\Lambda$ may still be preserved, in a way very similar to residual correlations. We call this effect a "feed-up" and also show it in Fig. 1.

In summary, the residual correlation mechanism produces a complicated web of interlinks between all measured and non-measured baryon pairs in the experiment. It is therefore not useful to analyze the measured baryon-baryon or baryon-antibaryon pair types separately. Instead a single fitting procedure must be created, where all those functions are analyzed simultaneously. In particular, they must use the same original correlation functions $C_{X Y}$ when calculating residual contributions to all measured correlation functions according to Eq. (3.1). This significantly complicates the procedure. However, it gives the opportunity to constrain the unknown interaction parameters even more, also for the pairs that are not measured directly but instead are only accessible via their residual contributions.

\section{References}

[1] T. Hell and W. Weise, Phys. Rev. C 90 (2014) no.4, 045801

[2] Y. Yamamoto, T. Furumoto, N. Yasutake and T. A. Rijken, Eur. Phys. J. A 52 (2016) no.2, 19

[3] M. Bleicher et al., J. Phys. G 25 (1999) 1859

[4] C. Patrignani et al. [Particle Data Group], Chin. Phys. C 40 (2016) no.10, 100001. 
[5] G. Alexander, U. Karshon, A. Shapira, G. Yekutieli, R. Engelmann, H. Filthuth and W. Lughofer, Phys. Rev. 173 (1968) 1452.

[6] J. A. Kadyk, G. Alexander, J. H. Chan, P. Gaposchkin and G. H. Trilling, Nucl. Phys. B 27 (1971) 13.

[7] B. Sechi-Zorn, B. Kehoe, J. Twitty and R. A. Burnstein, Phys. Rev. 175 (1968) 1735.

[8] C. J. Batty, Rept. Prog. Phys. 52 (1989) 1165.

[9] H. J. Pirner, B. Kerbikov and J. Mahalanabis, Z. Phys. A 338 (1991) 111.

[10] I. L. Grach, B. O. Kerbikov and Y. A. Simonov, Sov. J. Nucl. Phys. 48 (1988) 609 [Yad. Fiz. 48 (1988) 956].

[11] E. Klempt, F. Bradamante, A. Martin and J. M. Richard, Phys. Rept. 368 (2002) 119.

[12] V. G. J. Stoks and T. A. Rijken, Phys. Rev. C 59 (1999) 3009

[13] M. M. Nagels, T. A. Rijken and Y. Yamamoto, arXiv:1504.02634 [nucl-th].

[14] J. Haidenbauer and U. G. Meissner, Phys. Rev. C 72 (2005) 044005

[15] J. Haidenbauer, S. Petschauer, N. Kaiser, U.-G. Meissner, A. Nogga and W. Weise, Nucl. Phys. A 915 (2013) 24

[16] B. Abelev et al. [ALICE Collaboration], Phys. Rev. Lett. 109 (2012) 252301

[17] B. B. Abelev et al. [ALICE Collaboration], Phys. Rev. Lett. 111 (2013) 222301

[18] R. Lednicky, Phys. Part. Nucl. 40 (2009) 307

[19] R. Lednicky and V. L. Lyuboshits, Sov. J. Nucl. Phys. 35 (1982) 770 [Yad. Fiz. 35 (1981) 1316].

[20] A. Kisiel, H. Zbroszczyk and M. Szymański, Phys. Rev. C 89 (2014) no.5, 054916 